\title{
ERRATUM
}

Anna Iwaniec Hickerson · Derek Rinderknecht

Morteza Gharib

\section{Experimental study of the behavior of a valveless impedance pump}

Published online: 1 September 2005

(C) Springer-Verlag 2005

\section{Experiments in Fluids. DOl: 10.1007/s00348-005- 0946-z}

Due to an unfortunate error several characters in Table 1 did not appear correctly. The correct table is printed below.
Table 1 Parameters and measurements space

\begin{tabular}{llll}
\hline Parameter & Range & Step size & Accuracy \\
\hline Pinching frequence & $1-8 \mathrm{~Hz}$ & $0.1 \mathrm{~Hz}$ & $0.1 \mathrm{~Hz}$ \\
Pincher position & $2.5-12.7 \mathrm{~cm}$ & 1.3 & $0.3 \mathrm{~cm}$ \\
Transmural pressure & $0.24-0.69 \mathrm{kPa}$ & $0.22 \mathrm{kPa}$ & $0.1 \%$ \\
$\begin{array}{l}\text { Systemic resistance } \\
\text { Meterial }\end{array}$ & Qualitative & & \\
Duty cycle & Qatex, polyethylene & & \\
\hline Measurement & Ranglitative & & \\
\hline Flow rate & $\pm 8.3 \times 10^{-4} \mathrm{~m}^{3} / \mathrm{s}$ & $4.2 \times 10^{-8} \mathrm{~m}^{3} / \mathrm{s}$ \\
Pressure difference & $34.6 \mathrm{kPa}$ & $0.1 \%$ & \\
\hline
\end{tabular}

The online version of the original article can be found at http:// dx.doi.org/10.1007/s00348-005-0946-z

A. I. Hickerson $(\bowtie) \cdot$ D. Rinderknecht

Option of Bioengineering, California Institute of Technology, MC 205-45 1200 E California Blvd, Pasadena, CA, 91125 USA

E-mail: iwaniec@caltech.edu

M. Gharib

Hans W. Liepmann Professor of Aeronautics and Bioengineering, California Institute of Technology, MC 205-45 1200 E California Blvd, Pasadena, CA, 91125 USA 